\title{
Step-wise Behavior of Vortex-Lattice Melting Transition in Tilted Magnetic Fields in Single Crystals $\mathrm{Bi}_{2} \mathrm{Sr}_{2} \mathrm{CaCu}_{2} \mathrm{O}_{8+\delta}$
}

\author{
J. Mirkovića ${ }^{a, b}$, S.E. Savel'ev ${ }^{a}$, E. Sugahara ${ }^{a}$ and K. Kadowaki ${ }^{a}$ \\ ${ }^{a}$ Institute of Materials Science, The University of Tsukuba, 1-1-1 Tennodai, Tsukuba 305-8573, Japan, and \\ CREST, Japan Science and Technology Corporation (JST), Japan \\ ${ }^{b}$ Faculty of Sciences, University of Montenegro, PO Box 211, 81000 Podgorica, Montenegro, Yugoslavia
}

\begin{abstract}
The vortex lattice melting transition in single crystals $\mathrm{Bi}_{2} \mathrm{Sr}_{2} \mathrm{CaCu}_{2} \mathrm{O}_{8+\delta}$ was studied by the inplane resistivity measurements in magnetic fields tilted away from the $c$-axis to the $a b$-plane. In order to avoid the surface barrier effect which hinders the melting transition in the conventional transport measurements, we used the Corbino geometry of electric contacts. For the first time, the complete $H^{c}-H^{a b}$ phase diagram of the melting-transition in $\mathrm{Bi}_{2} \mathrm{Sr}_{2} \mathrm{CaCu}_{2} \mathrm{O}_{8+\delta}$ is obtained. The $c$-axis melting field component $H_{\text {melt }}^{c}$ exhibits the novel, step-wise dependence on the in-plane magnetic fields $H^{a b}$ which is discussed on the base of the crossing vortex lattice structure. The sharp change of resistance behavior observed near the $a b$-plane suggests transformation from first-order to second-order phase transition.
\end{abstract}

PACS numbers: 74.60.Ge, 74.60.Ec, 74.72.Hs

After discovery of high temperature superconductors, the phase diagram of the vortex state has been reconsidered because the novel vortex phases and phase transitions have been recognized [1]. Among them, the most intriguing phenomena is the first-order vortex-lattice melting transition (hereafter VLMT) [2], [3]. However, despite a lot of experimental and theoretical efforts, the nature of this phenomenon is not completely understood yet. In particular, it is not clear what happens with melting transition in highly anisotropic systems such as $\mathrm{Bi}_{2} \mathrm{Sr}_{2} \mathrm{CaCu}_{2} \mathrm{O}_{8+\delta}$ if magnetic field is tilted away from the $c$-axis, especially close to the $a b$-plane.

In contrast to melting transition in $\mathrm{YBa}_{2} \mathrm{Cu}_{3} \mathrm{O}_{7-\delta}$ 纤 described well by the anisotropic 3D Ginzburg-Landau (GL) theory [5], it was noticed [6] that VLMT in $\mathrm{Bi}_{2} \mathrm{Sr}_{2} \mathrm{CaCu}_{2} \mathrm{O}_{8+\delta}$ obeys neither 3DGL nor 2D [7] scaling. Later, Oii et al. [8] found that the $c$-axis melting field component $H_{\text {melt }}^{c}$ depends linearly on the in-plane magnetic field $H^{a b}$ in $\mathrm{Bi}_{2} \mathrm{Sr}_{2} \mathrm{CaCu}_{2} \mathrm{O}_{8+\delta}$. The authors explained the observed behavior through suppression of the Josephson coupling by the in-plane magnetic fields. They concluded that the anisotropy increases with $H^{a b}$ and should become infinite in a certain magnetic field at which the decoupling of the vortex lattice occurs. On the other hand, Koshelev [9] interpreted the linear dependence of $H_{\text {melt }}^{c}$ on $H^{a b}$ as an indication of the crossing lattice of Josephson vortices (JVs) and pancake vortex stacks (PVSs). According to this model, the linear dependence $H_{\text {melt }}^{c}\left(H^{a b}\right)$ breaks down and transforms into plateau as soon as the JV cores overlap. Such plateaulike behavior was observed recently [10,11], but there is still a question about the underlying mechanisms governing the melting transition in the tilted magnetic fields. In this regard, it is challenging to investigate what happens to the melting transition in much higher in-plane magnetic fields.

Here, we have investigated the melting transition using the in-plane resistivity measurements in the oblique magnetic fields in the whole angular range and found the dramatic new features, beyond pre-existing experimental data and theoretical models. The $H^{c}-H^{a b}$ phase diagram of melting transition exhibits the peculiar stepwise behavior. Besides, the transformation from the firstorder to the second-order phase transition near $a b$-plane is indicated.

It is worth to note that the novel experimental findings were obtained essentially by using the unconventional Corbino geometry of electric contacts in the resistivity measurements. As it is known well, the transport measurements in the conventional four probe strip geometry in $\mathrm{Bi}_{2} \mathrm{Sr}_{2} \mathrm{CaCu}_{2} \mathrm{O}_{8+\delta}$ [12,13 do not probe the true bulk properties of superconductor because surface barriers short-cut the current path and, as a consequence, the currents flow only near the sample edges. Therefore, the features of VLMT are also strongly hindered in resistivity measurements in the platelet samples. In order to avoid the smearing effect of VLMT and the nonlinear behavior in the vortex liquid phase due to surface barriers 14] we have used the Corbino electric contact geometry at which currents flow radially, far from the edges of sample (see inset of Fig. 1). Such experimental set-up considerably improved quality of resistivity data compared with conventional technique.

The in-plane resistivity measurements were performed for two as-grown single crystals 15] $\mathrm{Bi}_{2} \mathrm{Sr}_{2} \mathrm{CaCu}_{2} \mathrm{O}_{8+\delta}$ with transition temperature $T_{c}=90.3 \mathrm{~K}$ and $T_{c}=90.0$ $\mathrm{K}$ for samples \#S1 and \#S2, respectively. The diameters of the Corbino discs were $D=1.9 \mathrm{~mm}$ for \#S1 and $2.7 \mathrm{~mm}$ for \#S2, while the thickness was $t \approx 20 \mu \mathrm{m}$ for both samples. The resistance was measured by two pairs of electric contacts using the ac lock-in method at a low frequency of $37 \mathrm{~Hz}$. They agree with each other in a geometrical configuration error of about $5 \%$. The measurements of resistance were carried out as a function of the magnetic field $H$ at its different orientation $\theta$ with respect to the $c$-axis at various temperatures. The magnetic field was rotated by using $70 \mathrm{kOe}$ split coil with fine goniometer with angular resolution of $0.001^{\circ}$. 


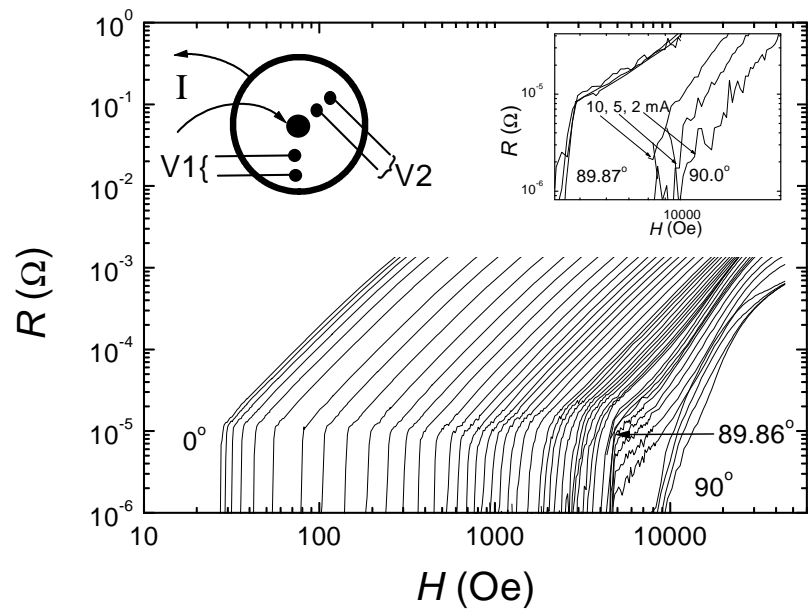

FIG. 1. The magnetic field dependence of the resistance measured at $T=85.2 \mathrm{~K}$ at various field orientations with respect to the $c$-axis in sample \#S1 with the current of $5 \mathrm{~mA}$. Left inset: a sketch of the sample with electric contacts in the Corbino geometry. Right inset: the magnetic field dependence of the resistance measured for two field orientations by three current levels.

The magnetic field dependence of the resistance at different orientations, which was measured at a particular temperature of $85.2 \mathrm{~K}$, is shown in Fig. 1. The steep resistance drop attributed to VLMT 16.17] is clearly detected even in oblique magnetic fields and, surprisingly, changes only weakly up to $\theta=89.86^{\circ}$. We should emphasize that the step height of the resistance anomaly practically remains the same and sharply separates the vortex solid and vortex liquid phases. As far as we know, the VLMT has never been found so close to the $a b$-plane. However, as the angle $\theta$ exceeds the critical value, only $0.14^{\circ}$ away from the $a b$-plane, the resistance level of the kink suddenly begins to decrease, while the sharpness of the anomaly gets even stronger. Then, the kink feature finally vanishes in the instrumental noise, and qualitatively new, smooth dependence $R(H)$ sets in. Instead of the quadratic field dependence of the resistance, found in the vortex liquid phase, $R(H)$ follows essentially the nonpower law in the narrow angle region near the $a b$-plane. In addition, it is remarkable that the Ohmic response of the resistance observed in the vortex liquid state at angles $\theta<89.96^{\circ}$ is replaced by the non-Ohmic behavior (see inset of Fig. 1). This peculiar resistance behavior may indicate the novel vortex phase in magnetic fields parallel to the $a b$-plane and the change of character of phase transition from first-order to second-order. Such experimental finding could be related to the scenario proposed earlier [18], which suggests transformation from the firstorder vortex lattice melting transition into the secondorder vortex-lattice — vortex-smectic phase transition in magnetic fields near the $a b$-plane.
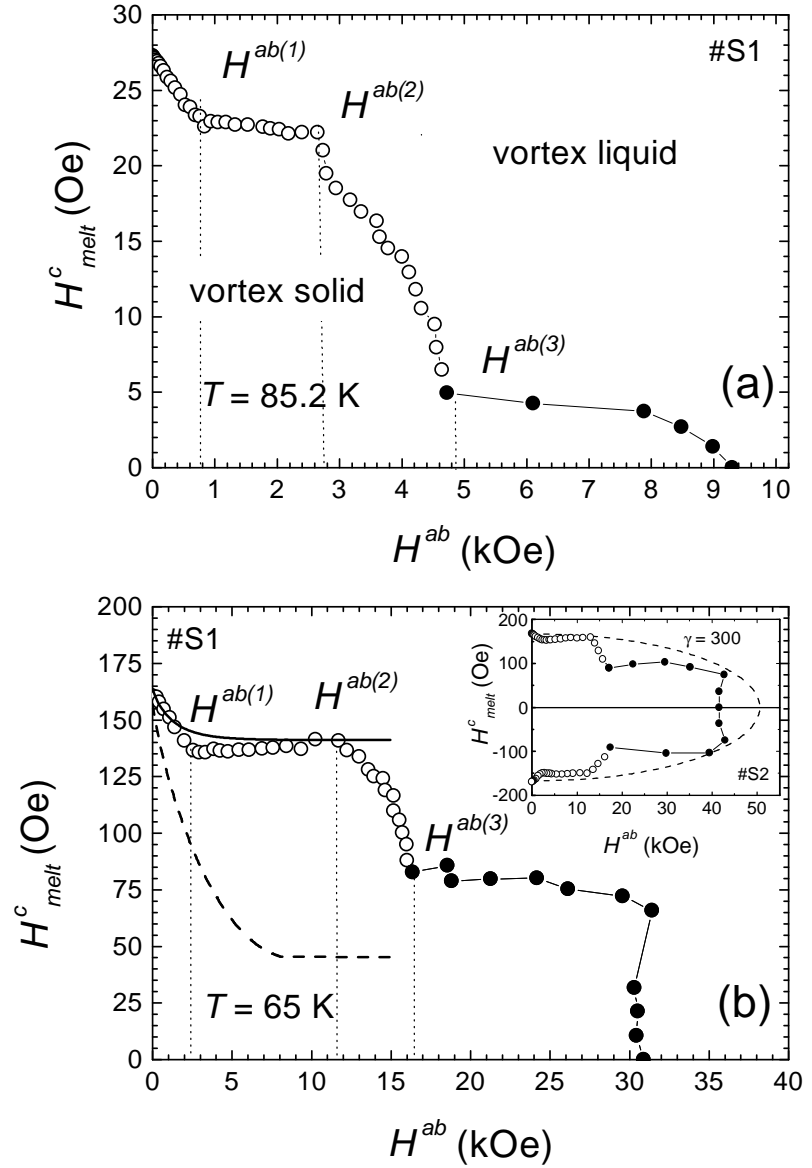

FIG. 2. Vortex lattice melting transition in the $H^{c}-H^{a b}$ plane for the sample \#S1 at two different temperatures of 85.2 K (a) and $65 \mathrm{~K}(\mathrm{~b})$. Dashed line in (b) is the fitting curve obtained from eq. (8) in [9]. The solid line is the fitting curve calculated after taking into account the interaction between PVs and currents of JVs. Inset in (b): $H^{c}-H^{a b}$ phase diagram for sample \#S2 at $T=62 \mathrm{~K}$. The solid line corresponds to the anisotropic 3D GL model with the anisotropy parameter of $\gamma=300$.

Using the resistance data obtained at two temperatures of $85.2 \mathrm{~K}$ and $65 \mathrm{~K}$ for the sample \#S1, the VLMT phase diagram is constructed in the $H^{c}-H^{a b}$ plane (Fig. $2)$. The melting transition was defined by the resistance criterion $R=10^{-6} \Omega$ in the whole angular range. The in-plane field dependence of the $c$-axis melting field component $H_{\text {melt }}^{c}$ exhibits a very intriguing step-wise behavior at both temperatures. At first stage, $H_{\text {melt }}^{c}$ decreases linearly with the in-plane magnetic field $H^{a b}$. Then, the linear dependence is abruptly terminated at field $H^{a b}{ }^{(1)}$ and transforms into plateau which continues to the field $H^{a b^{(2)}}$ where, surprisingly, the $c$-axis component of the 
melting field $H_{\text {melt }}^{c}$ starts to decrease again. This findin, is beyond the pre-existing experimental data and thec retical models. Finally, in the in-plane magnetic field above $H^{a b^{(3)}}$, the transition field $H_{\text {melt }}^{c}$ exhibits the sec ond weak in-plane field dependence without the resis tance kink anomaly (this region is indicated by filled sym bols in Fig. 2). The almost identical step-wise behavio of the melting transition is found also in the sample \#S2 as shown in the inset of Fig. $2 \mathrm{~b}$ at somewhat lower tem perature of $62 \mathrm{~K}$ [19].

The observed in-plane field dependence of the meltin transition is in the strong contrast with the $2 \mathrm{D}$ scalin 17. ruled only by the out-of-plane magnetic field compc nent. The inset in Fig. 2b demonstrates also the essen tial discrepancy between our data and the fitting curv based on the $3 \mathrm{D}$ scaling [5] $H_{\text {melt }}(\theta)=H_{\text {melt }}(0) /\left(\cos ^{2} \theta\right.$ $\left.\gamma^{-2} \sin ^{2} \theta\right)^{1 / 2}$, where $H_{\text {melt }}(0)$ is the melting field fo $\theta=0^{\circ}$ and $\gamma=300$ is chosen as the anisotropy param eter. Furthermore, the second decrease of the field $\mathrm{d} \epsilon$ pendence $H_{\text {melt }}^{c}\left(H^{a b}\right)$ after plateau can not be explaine in the frame of the phenomenological concept of "decou pled" vortex state proposed by Ooi et al. [8], since ther would not be any in-plane field dependence of the meltin transition above the decoupling field.

The first theoretical explanation of the unusual lin ear dependence $H_{\text {melt }}^{c}\left(H^{a b}\right)$ was given by Koshelev [9] who analyzed the crossing vortex lattice with interaction between pancake vortices (PVs) and JVs. Using the general thermodynamical equality of the free energies of vortex solid and vortex liquid phases at the melting transition, Koshelev obtained the linear dependence of the $c$-axis melting field component: $H_{\text {melt }}^{c}\left(H^{a b}\right)=$ $H_{\text {melt }}^{c}(0)-4 \pi \epsilon_{J} H^{a b} /\left(\Delta B \Phi_{0}\right)$, where $\epsilon_{J}$ is the energy of a JV in the presence of PV lattice, $\Delta B$ is the jump of the magnetic induction at the melting point, and $\Phi_{0}$ is the quantum of the magnetic flux [20]. In general, the energy of JV contains both, the self energy of JV, $\epsilon_{J}^{\text {self }}$ (eq. (3) in [9]), and the interaction energy $E_{P J}$ of PVS with currents generated by $\mathrm{JV}$, i.e. $\epsilon_{J}=\epsilon_{J}^{\text {self }}-E_{P J}$. The self energy contribution elevates the free energy of vortex solid phase and reduces the stability of the vortex crystal, while the second term acts in the opposite way, through decreasing of the free energy. The latter, interaction term, $E_{P J}$ was omitted in the analysis [9] of the melting transition for the case of a dense PV lattice. Figure $2 \mathrm{~b}$ shows the fitting curve (dashed line) obtained from the equation (8) of Reference [9] with rather high value of anisotropy parameter $\gamma=1500$ (the lower values of $\gamma$ give even stronger discrepancy with experimental data), the in-plane penetration depth $\lambda=2000 / \sqrt{1-T^{2} / T_{c}^{2}} \AA$, the interlayer distance $s=15 \AA$, and $\Delta B=0.35$ Oe. It is easy to see that the above mentioned model qualitatively describes our experimental data but the calculated average slope $\left(d H_{\text {melt }}^{c} / d H^{a b} \approx 0.025\right)$ as well as the expected breaking field $\left(H^{a b^{(1)}} \approx 7 \mathrm{kOe}\right)$ appeared to be several times higher than our experimental values ( $\left.d H_{\text {melt }}^{c} / d H^{a b} \approx 0.01, H^{a b(1)} \approx 2.5 \mathrm{kOe}\right)$. (a)

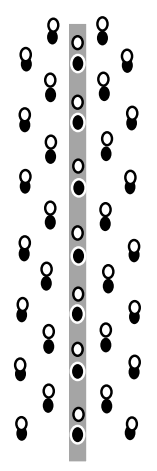

(b)

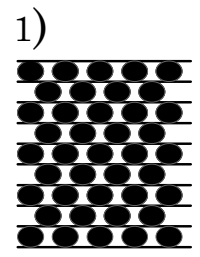

2)

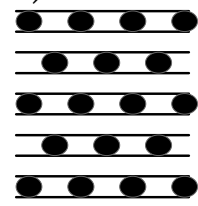

3)

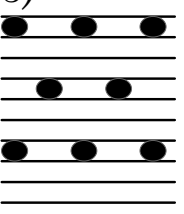

4)

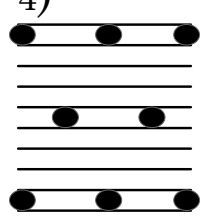

FIG. 3. a) The sketch of the PV lattice deformation due to the interaction between PVs and currents of JVs: filled circles correspond to the positions of PVs in an ideal lattice, open circles mark PVs in the presence of JV. b) Schematic pictures of the JV lattice with different periods along the $c$-axis.

On the other hand, in the experimentally studied magnetic field range and for the more realistic value of the anisotropic parameter $\gamma \approx 300$, the size of the nonlinear JV core $\lambda_{J}=\gamma s$ is about the same distance as the distance between pancakes vortices, $a_{p}$. In such a case, according to Koshelev [9], the interaction between PVSs and currents generated by JVs becomes important, and it is necessary to consider also the energy $E_{P J}$. Due to Lorentz force, PVs shift from their equilibrium positions (Fig. 3 a) reducing the free energy of the solid phase. Thus, the vortex crystal is stabilized and the $c$ axis melting field component decreases more slowly with the in-plane magnetic field. Therefore, the transformation of the linear dependence $H_{\text {melt }}^{c}\left(H^{a b}\right)$ into plateau could be related not to the overlapping of the JV cores, but to the compensation of the self energy of JVs by their interaction energy with PVSs. The rough calculations [21], taking into account the pinning of PVSs born by the interaction with currents of JVs, improve the correlation between model [9] and experimental data (Fig. $2 \mathrm{~b}$ (solid line)). The fitting curve was obtained under assumption that the pinning term is suppressed logarithmically by the in-plane field similar to the self-energy of Josephson vortex [9]. Nevertheless, this term decreases faster in higher in-plane fields due to diminishing of currents generated by JVs, when the distance $b_{J}$ between JVs along the $c$-axis approaches $2 s$. This means that the mentioned compensation can be destroyed in the field close to $\Phi_{0} / b_{J} a_{J}=\Phi_{0} / 2 \sqrt{3} \gamma s^{2}$ ( $a_{J}$ is the distance between JVs in a layer) and the new decay of the $c$-axis melting field component could follow again; for $\gamma=300$, $\Phi_{0} / 2 \sqrt{3} \gamma s^{2} \approx 9 \mathrm{kOe}$, which is close to the observed value $H^{a b^{(2)}}$ at $T=65 \mathrm{~K}$. Next, we point out that the disap- 
pearance of the kink anomaly at the field $H^{a b^{(3)}}$ could indicate the so-called "lock-in" transition [22] above which the observed behavior could be related to the melting of JV lattice via the second-order phase transition [18,23.

Finally, we note that the unusual step-wise behavior of the melting transition seems to be induced by the layer structure of $\mathrm{Bi}_{2} \mathrm{Sr}_{2} \mathrm{CaCu}_{2} \mathrm{O}_{8+\delta}$ which governs, in particular, the behavior of the JV lattice. According to Bulaevskii and Clem [24], the JV lattice undergoes the sequential structural first-order phase transitions between lattices with different periods. These transitions are based on the fact that the JVs are able to occupy only the space in-between the $\mathrm{CuO}_{2}$ planes, i.e. the distance $b_{J}$ between JVs along the $c$-axis should have discrete values $b_{J}=k s$, where $k$ is an integer. The structural transitions could explain the sharpness of dependence $H_{\text {melt }}^{c}\left(H^{a b}\right)$ at points $H^{a b^{(1)}}$ and $H^{a b^{(2)}}$ since the interaction between PVS and JVs is sensitive to the change of JV lattice parameters $b_{J}$ and $a_{J}$. Interestingly, the ratio of the magnetic fields where last two structural transitions happen (from the lattice 1 to 2 and from 2 to 3 in Fig. 3b) is $25 / 9$, being close to the experimentally observed value $H^{a b^{(2)}} / H^{a b^{(3)}} \approx 3-4$ at all temperatures. In summary, we present for the first time the complete $H_{\text {melt }}^{c}-H^{a b}$ phase diagram of VLMT in the single crystals $\mathrm{Bi}_{2} \mathrm{Sr}_{2} \mathrm{CaCu}_{2} \mathrm{O}_{8+\delta}$. In strong contrast to the conventional superconductors and $\mathrm{YBa}_{2} \mathrm{Cu}_{3} \mathrm{O}_{7-\delta}$, we observe the step-wise behavior of the melting transition, which reflects the layer structure of the system and could be related to interaction between pancake vortices and Josephson vortex lattice. The resistivity behavior indicates that melting transition changes its character from first-order to second-order phase transition in magnetic fields applied very close to the $a b$-plane.

We appreciate the enlightening discussions with A.E. Koshelev, J.R. Clem, M. Tachiki, G. Crabtree and A.I. Buzdin.

* On leave from All Russian Electrical Engineering Institute, 111250 Moscow, Russia

[1] For instance, G. Blatter et al., Rev. Mod. Phys. 66, 1125 (1994).

[2] D.R. Nelson and H.S. Seung, Phys. Rev. B39, 9153 (1989).

[3] H. Safar et al., Phys. Rev. Lett. 69, 824 (1992); E. Zeldov et al., Nature 375, 373 (1995); A. Schilling et al., Nature 382, 791 (1996); K. Kadowaki, Physica C236, 164 (1996).

[4] A. Schilling et al., Phys. Rev. Lett. 78, 4833 (1997).

[5] G. Blatter, V.B. Geshkenbein, and A.I. Larkin, Phys. Rev. Lett. 68, 875 (1992).

[6] B. Schmidt et al., Phys. Rev. B55, R8705 (1997).

[7] P.H. Kes et al., Phys. Rev. Lett. 64, 1063 (1990).

[8] S. Ooi, T. Shibauchi, N. Okuda and T. Tamegai, Phys.
Rev. Lett. 82, 4308 (1999).

[9] A.E. Koshelev, Phys. Rev. Lett. 83, 187 (1999).

[10] J. Mirković, E. Sugahara and K. Kadowaki, Physica B 284-288, 733 (2000).

[11] M. Konczykowski et al., cond-mat/9912284.

[12] D.T. Fuchs et al., Nature (London) 391, 373 (1998).

[13] J. Mirković, K. Kimura and K. Kadowaki Adv. in Supercond. XI (ISS'98), p. 557, Springer-Verlag Tokyo (1999).

[14] J. Mirković and K. Kadowaki, Physica B 284-288 759 (2000).

[15] T. Mochiku and K. Kadowaki, Trans. Mater. Res. Soc. Jpn. 19A, 349 (1993).

[16] S. Watauchi et al., Phys. C259, 373 (1996).

[17] J. Mirković, K. Kimura and K. Kadowaki, Phys. Rev. Lett. 82, 2374, (1999).

[18] L. Balents and D.R. Nelson, Phys. Rev. B52, 12951 (1995).

[19] We emphasize that our experimental results agree well to ac magnetization measurements over the common measured interval (for $H^{a b}<H^{a b(2)}$ compare 10 and [1]). However, in contrast to ac and dc magnetization technique 66, 8, 11,25], the resistance measurements with Corbino geometry do not lose sensitivity for fields aligned near the $a b$-plane, and thus, make it possible to get the complete $H_{\text {melt }}^{c}-H^{a b}$ phase diagram in the whole angular range.

[20] The general thermodynamical equation at the melting transition is: $F_{s}^{c}\left(B_{m 0}^{c}+\delta B_{m}^{c}\right)+\delta F_{s}=F_{l}^{c}\left(B_{m 0}^{c}+\delta B_{m}^{c}\right)+$ $\delta F_{l}$, where $F_{s}^{c}$ and $F_{l}^{c}$ are the free energies of the vortex solid and vortex liquid states for the $c$-axis magnetic field, respectively, $B_{m 0}^{c}$ is the melting field in $H^{a b}=0$, $\delta B_{m}^{c}$ is the shift of the $c$-axis melting field induced by $H^{a b}$, while $\delta F_{s}$ and $\delta F_{l}$ are the contributions to the free energies born by the in-plane magnetic field. Expanding $F_{s}^{c}$ and $F_{l}^{c}$ with respect to $\delta B_{m}^{c}$, one gets $B_{m}^{c}=$ $B_{m 0}^{c}-4 \pi\left(\delta F_{s}-\delta F_{l}\right) / \Delta B$. In the case of crossing lattice, $\delta F_{s}=\left(B^{a b}\right)^{2} / 8 \pi-\epsilon_{J} B^{a b} / \Phi_{0}$, i.e. it consists of the magnetic energy and the energy of JVs in the presence of PV lattice. Taking into account that Josephson coupling is suppressed in the vortex liquid state $\left(\delta F_{l} \approx\left(B^{a b}\right)^{2} / 8 \pi\right)$, and neglecting the difference between magnetic induction and external magnetic field, we obtain the equation as presented in the text.

[21] The pinning contribution to the free energy of the vortex solid phase is obtained from the condition that the gain of energy $E(x)$, born by interaction between PVS and currents of JV, depends on the distance $x$ between PVS and JV: the maximum of $E(x)$ corresponds to PVS placed on the center of JV. For the PV lattice with the intervortex distance $a_{p}=\sqrt{2 \phi_{0} / \sqrt{3} H^{c}}$, the pinning energy $E_{P J}$ per unit length of JV can be evaluated as $E_{P J}=\left(E(0)-E\left(a_{p} / 2\right)\right) / a_{p} \approx A E_{\times} a_{p} / \lambda_{J}^{2}$, where $E_{\times}=E(0)$ is defined by eq. (9) in [9], and $A$ is the coefficient close to unity (we used $A=0.5$ ). The corresponding contribution to the slope $d H_{\text {melt }}^{c} / d H^{a b}$ is $4 \pi A E_{\times} a^{p} /\left(\Phi_{0} \lambda_{J}^{2} \Delta B\right)$.

[22] D. Feinberg and C. Villard, Phys. Rev. Lett. 65, 919 (1990).

[23] Xiao Hu and Masashi Tachiki, cond-mat/0003068.

[24] L. Bulaevskii and J.R. Clem, Phys. Rev. B 44, 10234 (1991).

[25] K. Kadowaki and K. Kimura, Phys. Rev. B57, 11674 (1998). 